\title{
CFD-modelling of free surface flows in closed conduits
}

\section{Katharina Teuber* and Tabea Broecker}

Chair of Water Resources Management and Modeling of Hydrosystems, Technische Universität Berlin, Gustav-Meyer-Allee 25, 13355 Berlin, Germany

Email: katharina.teuber@uwi.tu-berlin.de

Email: tabea.broecker@uwi.tu-berlin.de

*Corresponding author

\section{Arnau Bayón}

Research Institute of Water and Environmental Engineering (IIAMA),

Universitat Politècnica de València,

C. de Vera, s/n, 46022 - València, Spain

Email: arbarbar@iiama.upv.es

\section{Gunnar Nützmann}

Leibniz-Institute of Freshwater Ecology and Inland Fisheries, Müggelseedamm 310, 12587 Berlin, Germany

and

Geography Department, Humboldt-University of Berlin, Unter den Linden 6, 10099 Berlin, Germany

Email: nuetzmann@igb-berlin.de

\section{Reinhard Hinkelmann}

Chair of Water Resources Management and Modeling of Hydrosystems, Technische Universität Berlin, Gustav-Meyer-Allee 25, 13355 Berlin, Germany Email: reinhard.hinkelmann@wahyd.tu-berlin.de

\begin{abstract}
Computational fluid dynamics (CFD) is gaining an increasing importance in the field of hydraulic engineering. This publication presents different application examples of a two-phase approach as implemented in the open source software OpenFOAM. The chosen approach is based on the volume of fluid method focusing on the simulation of flow in closed conduits. Three examples are presented: single-phase flow over a ground sill and free surface flow over a hill as well as complex free surface flow in a sewer model. The first example compares the results of different RANS turbulence models with experimental results. The results of the second example are compared with an analytical solution. In the last example the behaviour of the free surface flow is compared with the results of a model test and existing simulations using a simplified, open channel geometry for the closed conduit. For the examples analysed, the two-phase approach provides stable and reliable results.
\end{abstract}

Keywords: computational fluid dynamics; CFD; three-dimensional models; turbulence simulation and modelling; volume of fluid.

Reference to this paper should be made as follows: Teuber, K., Broecker, T., Bayón, A., Nützmann, G. and Hinkelmann, R. (2019) 'CFD-modelling of free surface flows in closed conduits', Progress in Computational Fluid Dynamics, Vol. 19, No. 6, pp.368-380.

Biographical notes: Katharina Teuber received her BSc in Civil Engineering from the TU Berlin, Germany in 2012 and MSc in Civil Engineering from the TU Berlin, Germany in 2015. Since 2015, she is a PhD student at TU Berlin at the Chair of Water Resources Management and Modeling of Hydrosystems.

Tabea Broecker received her BSc in Civil Engineering from the TU Berlin, Germany in 2011 and MSc in Civil Engineering from the TU Berlin, Germany in 2014. Currently, she is a $\mathrm{PhD}$ student at TU Berlin at the Chair of Water Resources Management and Modeling of Hydrosystems. 
Arnau Bayón obtained his MSc in Hydraulic Engineering and Environment from the Universitat Politécnica de Valéncia, Spain, and MSc in Fluid Mecahnics from the Von Karman Institute for Fluid Dynamics, Belgium. He conducted his $\mathrm{PhD}$ on numerical/experimental modelling of hydraulic structures. He has been a Visiting Researcher at Instituto Superior Técnico de Lisboa, Portugal. Currently, he is an Assistant Professor at the Department of Hydraulic Engineering and Environment at Universitat Politécnica de Valéncia.

Gunnar Nützmann received his Diploma in Numerical Mathematics in 1973 in Rostock, and Doctoral in Finite Element Simulation of Variable Saturated Flow in Porous Media in 1981 in Berlin. He was a Senior Scientist at the Academy of Science in Berlin (Institute of Mechanics, Institute of Geography and Geoecology), and from 1992 on at the Leibniz-Institute of Freshwater Ecology and Inland Fisheries (IGB). His habilitation was in 1998 at Technical University of Berlin. Since 2002 he holds a Professorship in Hydrology at the Geography Department, Humboldt-University of Berlin and he is the head of the Department of Ecohydrology at the IGB. His research interests include numerical modelling of soil- and groundwater flow and transport with the special consideration of exchange processes between surface water and subsurface water systems.

Reinhard Hinkelmann received his Diploma in Civil Engineering 1991 and Doctoral in Shallow Water Modelling/High Performance Computing in 1997 both at Hannover University, Germany. He was a Senior Engineer at TU Braunschweig, Germany 1997-2000 and at Stuttgart University, Germany 2000-2004 and made a Habilitation in efficient numerical methods for surface and subsurface flow and transport processes/hydroinformatics in 2002. Since 2004 he is a Professor for Water Resources Management and Modelling of Hydrosystems, TU Berlin, Germany. His research interests include modelling of flow and transport processes in surface water, subsurface systems (groundwater, two-phase flow in porous media), and hydroinformatics.

\section{Introduction}

Numerical multiphase solvers have gained an increasing importance in the field of hydraulic research. In this paper, the open field operation and manipulation (OpenFOAM) solver interFoam (Rusche, 2002) which is based on a VOF interface capturing approach (Section 2.2) will be analysed.

In a number of recent publications, such as Bayón et al. (2015), Bayón and López-Jiménez (2015), Schulze and Thorenz (2014) and Thorenz and Strybny (2012) the interFoam solver was used to analyse complex hydraulic cases in open channels. Possible application areas are inland waters (Thorenz and Strybny, 2012), coastal areas (Higuera et al., 2013) as well as sewer systems (Bayón et al., 2015). The previously mentioned publications simulated the water phase behaviour and used the two-phase approach in order to display the movement of the free water surface.

Bayón and López-Jiménez (2015) used the interFoam solver to analyse a hydraulic jump in a rectangular channel with smooth walls. A comparison of different variables with experimental results led to the conclusion that the model can be applied to real-life cases of designing hydraulic structures. In Bayón et al. (2015), the test case of the hydraulic jump was extended to a more complicated surrounding consisting of an existing sewer stretch with different hydraulic structures such as weirs, quickly-varying shapes, macro-roughness elements, fast and slow flow regimes as well as hydraulic jumps. The results were compared with experimental results from a 1:20 scale model and also showed a good agreement. In our last example we will use this sewer geometry. Due to stability problems, the sewer has been simulated as an open channel with an atmospheric top boundary.

The novelty applied to the existing setup is the closed system setup by defining a closed (wall) top boundary instead of an open atmospheric top boundary as it has been used in Bayón et al. (2015) and a different definition of the outlet boundary condition.

The importance of the correct choice of a turbulence model was shown in Bayón et al. (2015) and Bayón and López-Jiménez (2015). A sensitivity analysis in Bayón and López-Jiménez (2015) showed a good performance of the Standard k- $\epsilon$ turbulence model.

A number of pipe flow simulations have already been performed applying the VOF approach in closed ducts. A few of them shall be outlined in the following and their certain characteristics shall be pointed out.

Shuard et al. (2016) compared simulations of two-phase flow in a circular pipe using the interFoam solver with results of a mechanistic model. Similar simulations have been carried out by Thaker and Banerjee (2013) analysing the transition between different flow regimes as well as the development of flow regimes. The results were compared to experimental measurements using similar boundary conditions. The boundary conditions that have been used by Shuard et al. (2016) have been used for a different area of application by Kinyua et al. (2016). Here, tubular anaerobic digesters have been modelled by including tracer simulations. The simulations carried out by Shuard et al. (2016), Thaker and Banerjee (2013) and 
Kinyua et al. (2016) analysed flow behaviour in closed ducts. By applying a constant value as an outlet pressure boundary condition, a free outflow out of the domain was achieved, assuming that the water level is not impounded by any downstream water level.

This short literature review shows that computational fluid dynamics (CFD) models are an upcoming topic in hydraulic engineering but the simulation results strongly depend on factors such as boundary conditions, mesh quality and turbulence models. Existing validation shows a good accuracy of the interFoam solver with experimental data for open systems with atmospheric top boundaries and for closed ducts with free outflows, however, there is a lack of research on the behaviour of free surface flows in closed ducts with a defined water level. In this paper we want to investigate simulations that describe free surface flow in closed pipes where at the same time a certain outlet water level is desired (see also Bayón et al., 2015). This problem occurs in cases where the area of interest comprises a large system, i.e., a complete sewer system. Due to limitations of computational resources it is usually not feasible to simulate the whole system, however, it is possible that the water level at the end of the stretch that is being considered in the simulation influences the water level within the model domain. Therefore it is not possible to use a free outflow as it has been used in previous studies. The literature analysis shows that these systems have not been addressed in previous publications.

The simulation of closed systems is interesting for different areas of application, i.e., for the modelling of in-sewer processes (Edwini-Bonsu and Steffler, 2004; Gessner et al., 2014; Hvitved-Jacobsen et al., 2013; Rootsey et al., 2012). A detailed analysis of the air phase behaviour will be subject to future research. To ensure a correct behaviour of the water phase, a thorough validation of flow simulations in closed conduits using the solver interFoam is performed in three steps. First, the behaviour of the water phase is analysed by comparing the numerical data of flow over a hill for single-phase flow with experimental results by Almeida et al. (1993). The results of the first test case show us which turbulence model is most suitable for the problems analysed. Then, the different outlet boundary conditions are compared using a simple two-phase simulation. The interface behaviour is analysed by comparing the simulated results of water level drawdown due to a ground sill with analytical results based on continuity and Bernoulli's equation. In a last step, the stability and the accuracy in describing a practical test case is checked by simulating a complex sewer geometry (Bayón et al., 2015).

The paper is organised as follows: Section 2 gives an overview over the methods and materials. The computational test cases are presented in Section 3. The validation of the different parts is done as follows: single phase water flow over a ground sill, free surface flow over a hill, complex free surface flow in a sewer model. In Section 4 , the results of the validation are summarised.

\section{Methods and materials}

\subsection{Geometry and mesh}

Unstructured meshes created in the open source mesh generation tool gmsh were used for the single phase validation case (see Section 3.1) and for the free surface flow over a hill (see Section 3.2). The complex sewer case of Section 3.3 was discretised with a structured grid using the OpenFOAM utility snappyHexMesh. The detailed geometry of the different test cases will be outlined in the subsequent sections.

\subsection{Numerical model}

The open source CFD software OpenFOAM version 2.4.0 was used to simulate different test cases. Single and two-phase flow is calculated by using the solver interFoam based on a VOF approach. interFoam is a multiphase solver for immiscible and isothermal fluids that solves the three-dimensional Navier-Stokes equations using the finite-volume-method in space and the finite-differences-method in time. OpenFOAM allows parallel computations on a theoretically unlimited number of processor cores (Keough, 2014).

The conservation of mass [equation (1)] and momentum [equation (2)] for incompressible flow can be written as:

$$
\begin{aligned}
& \nabla \cdot \mathbf{U}=0 \\
& \frac{\partial \rho \mathbf{U}}{\partial t}+\mathbf{U} \cdot \nabla \rho \mathbf{U}=-\nabla p+\mu \Delta \mathbf{U}+\rho \mathbf{g}
\end{aligned}
$$

The viscosity term $\mu$ referred to in equation (2) contains the physical viscosity $\mu_{\text {phys }}$ as well as the turbulent viscosity $\mu_{\text {turb }}$ which will be obtained by a turbulence model:

$$
\mu=\mu_{\text {phys }}+\mu_{\text {turb }}
$$

The VOF method used in the interFoam solver uses a specific pressure formulation where $p_{r g h}$ is a modified pressure which is used in order to avoid the occurrence of steep pressure gradients caused by hydrostatic effects. In the following, $p_{\text {rgh }}$ will be referred to as:

$$
p_{r g h}=p-\rho \cdot \mathbf{g} \cdot \mathbf{h}
$$

The two immiscible fluids liquid and gas "are considered as one effective fluid throughout the domain, the physical properties of which are calculated as weighted averages based on the distribution of the liquid volume fraction, thus being equal to the properties of each fluid in their corresponding occupied regions and varying only across the interface" (Berberović et al., 2009), leading to a definition of $\rho$ and $\mu$ as follows:

$$
\begin{aligned}
& \rho=\alpha \rho_{\text {water }}+\rho_{\text {air }}(1-\alpha) \\
& \mu=\alpha \mu_{\text {water }}+\mu_{\text {air }}(1-\alpha)
\end{aligned}
$$


The $\alpha$ transport equation - also known as VOF equation - is used with an advanced formulation which can be considered as an evolution equation for the phase fraction $\alpha$ (Berberović et al., 2009):

$$
\frac{\partial \alpha}{\partial t}+\nabla \cdot(\alpha U)+\nabla \cdot\left((1-\alpha) U_{r} \alpha\right)=0
$$

"where $U_{r}=U_{g}-U_{l}$ is the vector of relative velocity, designated as the "compression velocity"'(Berberović et al., 2009). A detailed derivation of the equations can be found in Márquez Damián (2012).

In the equations above, $\mathbf{U}$ represents the ensemble averaged velocity field shared by the two fluids throughout the flow domain $[\mathrm{m} / \mathrm{s}] ; \rho$ is the density $\left[\mathrm{m}^{3} / \mathrm{s}\right] ; \mathrm{t}$ is time [s]; $\mathrm{p}$ is pressure $[\mathrm{Pa}] ; \mu_{p h y s}$ and $\mu_{\text {turb }}$ are the physical and turbulent viscosity $\left[\mathrm{Ns} / \mathrm{m}^{2}\right] ; \mathbf{g}$ is the acceleration vector due to gravity $\left[\mathrm{m} / \mathrm{s}^{2}\right] ; \mathbf{h}$ is a spatial position vector $[\mathrm{m}] ; \alpha$ is the volume fraction or indicator function $[-] ; U_{r}$ is the relative velocity between the phases $[\mathrm{m} / \mathrm{s}]$; the subscripts $l$ and $g$ denote different fluids liquid (water) and gas (air). The indicator function $\alpha$ is defined as:

$$
\alpha= \begin{cases}1 & \text { for a point inside fluid water } \\ 0<\alpha<1 & \text { for a point in the transitional region } \\ 0 & \text { for a point inside fluid air }\end{cases}
$$

The water surface is defined as the transition area where $\alpha=0.5$. The solver can be used as well for single-phase flow simulations. The volume fraction $\alpha$ is then 1 and constant over the whole domain.

Since the relative velocity cannot be computed directly from the one-fluid formulation in OpenFOAM, the numerical implementation of the relative velocity is as follows (Cifani et al., 2016):

$$
U_{r}=n_{\alpha} \min \left[C_{\alpha} \frac{|\phi|}{\left|S_{\alpha}\right|}, \max \left(\frac{|\phi|}{\left|S_{\alpha}\right|}\right)\right]
$$

where $n_{\alpha}$ is the normal vector of the cell surface, $\phi$ is the mass flux, $S_{\alpha}$ is the cell surface area, $C_{\alpha}$ is an adjustable coefficient on which the level of compression depends. The maximum of $U_{r}$ is bounded to the maximum face velocity in the flow field and the direction is aligned with $n_{\alpha}$ (Cifani et al., 2016; Hoang et al., 2013).

Equations (1) and (2) are solved by using the PIMPLE algorithm. As outlined in Bayón et al. (2015), pressure-velocity coupling is done by combining the inner corrector loops of the pressure implicit with splitting of operators (PISO) algorithm (Issa, 1985) with outer corrector loops of the semi-implicit method for pressure-linked equations (SIMPLE) algorithm (Patankar and Spalding, 1972). Detailed information on the algorithms can be found in Jasak (1996). As advection schemes, a total variation diminishing scheme (interGamma) (Jasak, 1996) combined with a flux corrected transport approach multidimensional limiter for explicit solutions (MULES) (Márquez Damián, 2013) is used.
Simulations were carried out until a quasi-steady state was reached. This quasi-steady state has been determined by sampling variables of interest such as the pressure and the velocity at different points in the water and air phase.

In the first example, different RANS turbulence models, namely the standard $\mathrm{k}-\varepsilon$ (Launder and Sharma, 1974), $\mathrm{k}-\omega$ (Wilcox, 1988) and k- $\omega$ shear stress transport (SST) (Menter, 1993, 1994) are compared. Wall functions have been used to describe the near-wall turbulent flow. It was ensured that the $y^{+}$-values for each simulation were in a range between 30 and 300 to guarantee the applicability of the wall functions.

\subsection{Boundary conditions}

Different boundary conditions were defined for single and two-phase simulations. The definition of sidewalls was similar for single and two-phase simulations but varied depending on the fact whether two or three-dimensional model setups were used. For two-dimensional test cases, so-called empty boundary conditions, which are implemented in OpenFOAM to describe sidewalls of two-dimensional geometries, were used. They declare that the sidewalls do not constitute solution directions within the defined domain. The sidewalls of three-dimensional testcases were defined by no-slip conditions. The detailed set of boundary conditions is explained in the chapters containing the respective test cases. However, a short description about the definition of outlet boundary conditions in free surface CFD models shall be given in the following.

In general, the definition of the outlet boundary condition in OpenFOAM using the interFoam solver differs from the definition in shallow-water models. In these models, often a water level is fixed at the outlet of the domain. In OpenFOAM, such a definition of a constant water level is not possible, therefore alternatives have to be looked for. Possibilities described in recent publications are to fix the pressure and the phase fraction value at the outlet (Thorenz and Strybny, 2012) or to specify a velocity profile at the outlet. A third option which circumvents the direct definition of a boundary condition in order to ensure a certain water level is to set an atmospheric pressure condition at the outlet and integrate a weir in the geometry close to the outlet (Bayón and López-Jiménez, 2015).

In this paper, a certain water level $\mathrm{h}$ at the outlet has been obtained in two different ways which will be referred to in the respective sections:

- $\quad$ Pressure boundary condition: $p_{r g h}$ is defined as a stepwise function at the outlet. The built-in function 'setFields' is used to overwrite uniform values at the outlet which is initially defined using a 'calculated' boundary condition: 
$p_{\text {rgh }}= \begin{cases}0 & \text { if } \mathrm{z}<\mathrm{h} \\ \max (\rho g h) & \text { if } \mathrm{z} \geq \mathrm{h}\end{cases}$

After the writing process, the type of boundary condition is changed to 'fixedValue'. The velocity at the outlet is defined as 'inletOutlet' condition which applies a null Neumann boundary condition in case of positive flux out of the domain and a user-specified fixed velocity in case of negative flux into the domain. In case of negative flux, the velocity is set to $\mathrm{U}=\left(\begin{array}{lll}0 & 0 & 0\end{array}\right)$. The phase fraction value $\alpha$ is defined as null Neumann boundary condition.

- Integration of weir: a weir structure in close proximity to the outlet is used as a means to maintain the water level in the domain. At the outlet, a constant pressure is defined using a Dirichlet boundary condition, the velocity is defined using the 'inletOutlet' condition. The phase fraction value $\alpha$ is specified using a null Neumann boundary condition.

In order to avoid stability problems, pressure boundary conditions need an accurate definition of initial conditions, i.e., the initial water level, flow velocity and pressure. Therefore, for simulations using this set of boundary conditions, suitable initial conditions have to be specified. They will be further outlined in the subsequent sections. Depending on the RANS turbulence model, an additional definition of boundary conditions for turbulent properties such as the turbulent kinetic energy $\mathrm{k}$ and the turbulent dissipation $\varepsilon$ for $\mathrm{k}-\varepsilon$ or the turbulent kinetic energy $\mathrm{k}$ and the specific dissipation $\omega$ for $\mathrm{k}-\omega$ models is needed depending on the chosen model. For the boundary condition, an initial guess of the turbulent properties has to be defined. During the simulation, these values are rewritten according to the simulation results.

\section{Applications}

\subsection{Single phase water flow over a ground sill}

In the first example, single-phase water flow over a two-dimensional model hill in a duct has been simulated and compared with experimental results obtained by Almeida et al. (1993).

Experiments were conducted in a two-dimensional duct with a height of $h_{\max }=0.17 \mathrm{~m}$ bounded by an upper and lower wall with a polynomial-shaped obstacle on its bottom (see Figure 1). The mean centreline velocity at the inlet amounted to $U_{0}=2.147 \mathrm{~m} / \mathrm{s}$ causing pressurised flow throughout the domain. Velocity profiles of the simulations in $\mathrm{x}$ and $\mathrm{y}$-direction at four (velocities in $\mathrm{x}$-direction) respectively two (velocities in $\mathrm{y}$-direction) different locations were compared to the measurements: $x_{-03}=-0.30 \mathrm{~m}$ (in front of the hill), $x_{00}=0.00 \mathrm{~m}$ (top of the hill), $x_{01}=0.03 \mathrm{~m}$ (end of the hill) and $x_{02}=$ $0.05 \mathrm{~m}$ (middle of recirculation zone). Figure 1 shows the model domain including the mesh that has been used and the different measurement stations. Simulations have been carried out using different RANS turbulence models (standard $\mathrm{k}-\varepsilon, \mathrm{k}-\omega$, and $k-\omega \mathrm{SST}$ ).

A grid convergence study following Celik et al. (2008) has been conducted in order to ensure mesh independence. Five different meshes with different cell sizes were tested using the flow velocity in x-direction $U_{x}$ in 15 locations in the nearfield of the hill structure. The cell sizes and total number of cells of the analysed meshes were $0.0293 \mathrm{~m}(69,326$ cells $), 0.0346 \mathrm{~m}$ (31,102 cells), $0.0416 \mathrm{~m}$ (15,319 cells), $0.0495 \mathrm{~m}(7,338$ cells $)$, and $0.0581 \mathrm{~m}$ $(3,855$ cells), leading to a global refinement ratio between meshes of 2, which is above the minimum recommended value of 1.3 (Celik et al., 2008). Figure 2(a) shows the resulting velocities of three indicator locations $\phi_{4}, \phi_{7}$ and $\phi_{10}$. The position of these three points is displayed in Figure 1. Convergence was reached between the second finest and finest mesh. The computed RMSE for the different refinement steps (i.e., step 1: between coarsest and second coarsest mesh) is displayed in Figure 2(b). It shows a significant decline between the third and fourth refinement step to $5.5 \mathrm{e}-5 \mathrm{~m} / \mathrm{s}$. These results lead to the conclusion that the second finest mesh analysed has reached grid independence, so all subsequent analysis is conducted on the mesh of $0.0346 \mathrm{~m}$ element size and a total number of 31,102 cells. For the selected mesh, the average apparent order as defined by Celik et al. (2008) is 1.9, very close to the model formal order. A variable time step in dependence of the Courant number has been used, which converged to approximately $\Delta \mathrm{t}=0.0007 \mathrm{~s}$. The model convergence to a quasi-steady state can be observed after a few seconds. All simulations have been carried out with a simulation time of ten seconds. For all simulations, Intel Xeon IvyBridge E5-2695v2 cores have been selected. Using parallel computations on 16 cores led to computation times of approximately 34 minutes. In order to reach a good agreement with the experimental setup, a velocity profile using the experimental data has been imposed as an inlet boundary condition. The upper and lower walls were specified with no-slip conditions. At the sidewalls, empty boundary conditions have been applied. At the outlet, the water level was defined using a constant $p_{r g h}$-value defined by the maximum hydrostatic pressure: $p_{\text {rgh }}=\max (\rho g h)$. As the initial condition, the domain is filled with water but no velocities are predefined. The velocity profile therefore develops during the simulation. 
Figure 1 Domain of single-phase, two-dimensional hill flow including measurement locations, three selected indicator locations for grid convergence study and computational mesh

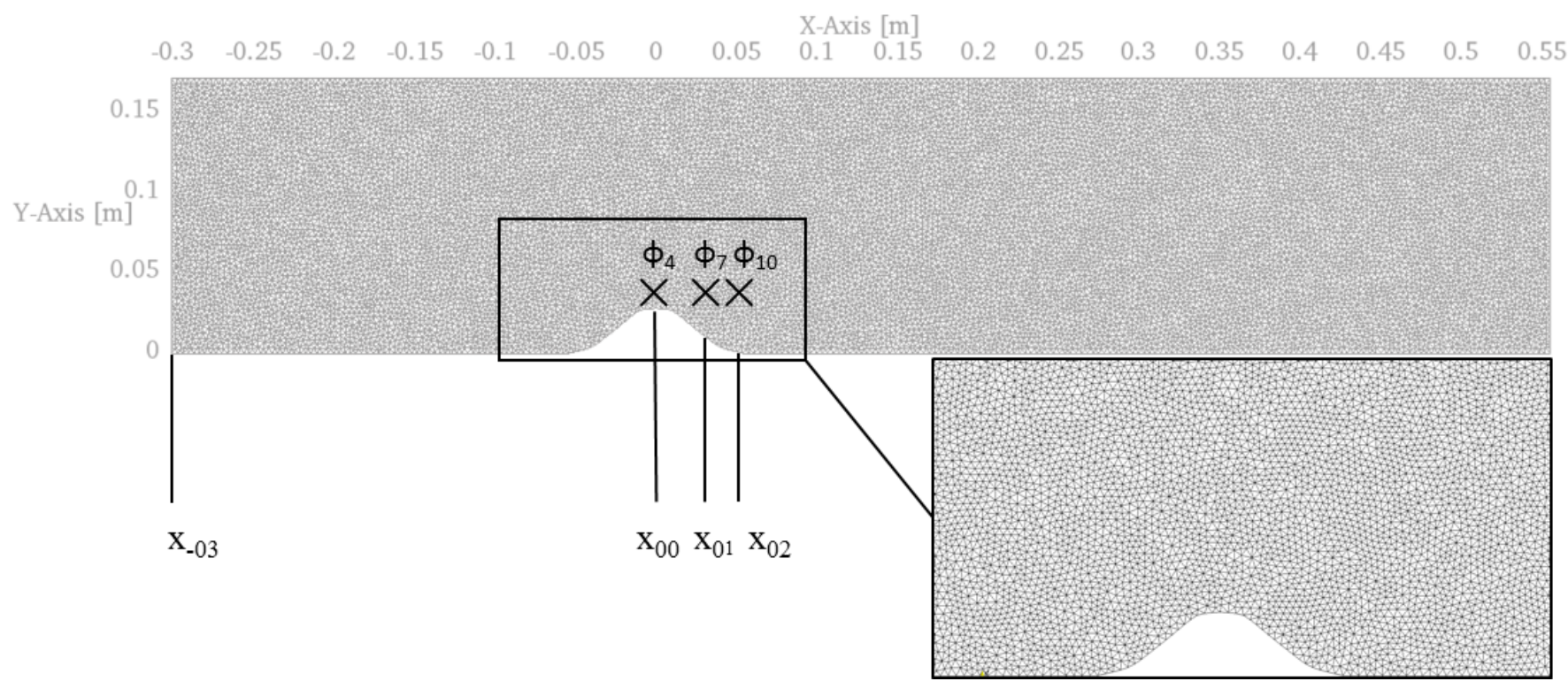

Source: Davroux et al. (1995)

Figure 2 Mesh sensitivity analysis, (a) comparison of simulated indicator values $\phi$ (flow velocities) for different grid sizes in three points within the domain (b) RMSE between different refinement steps over all 15 indicator values

a) Velocities

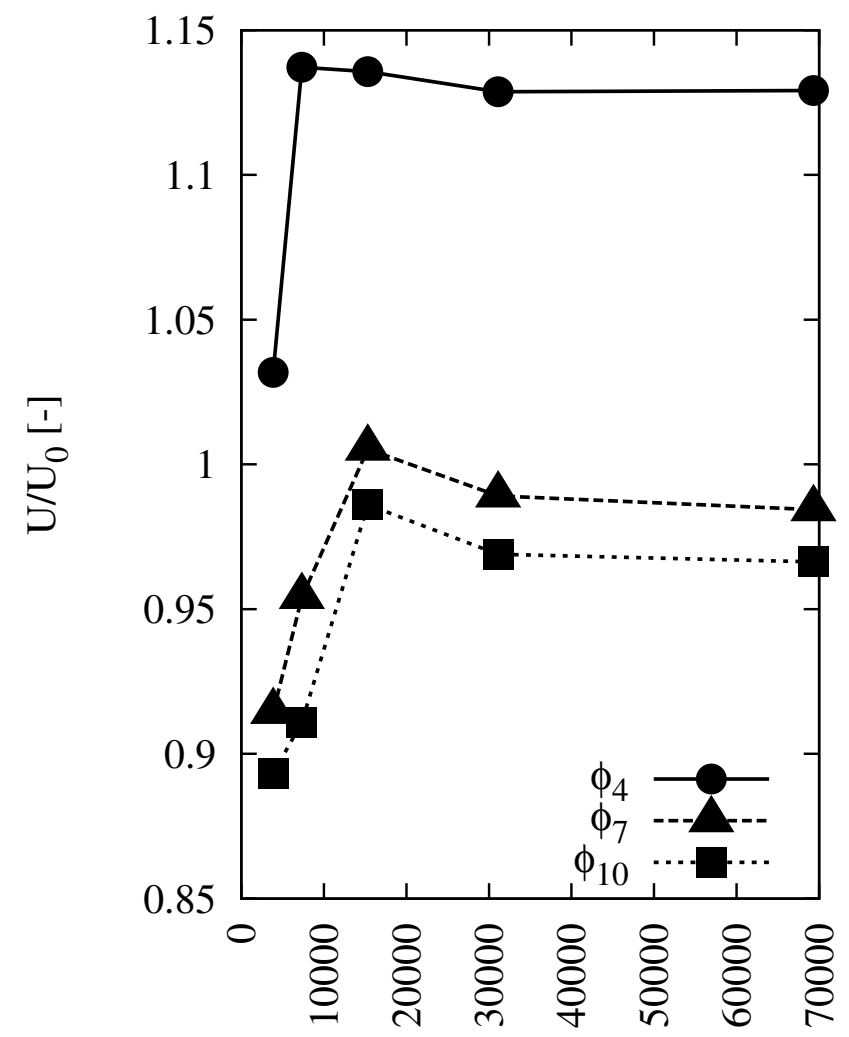

No. of cells [-]

\section{b) RMSE}

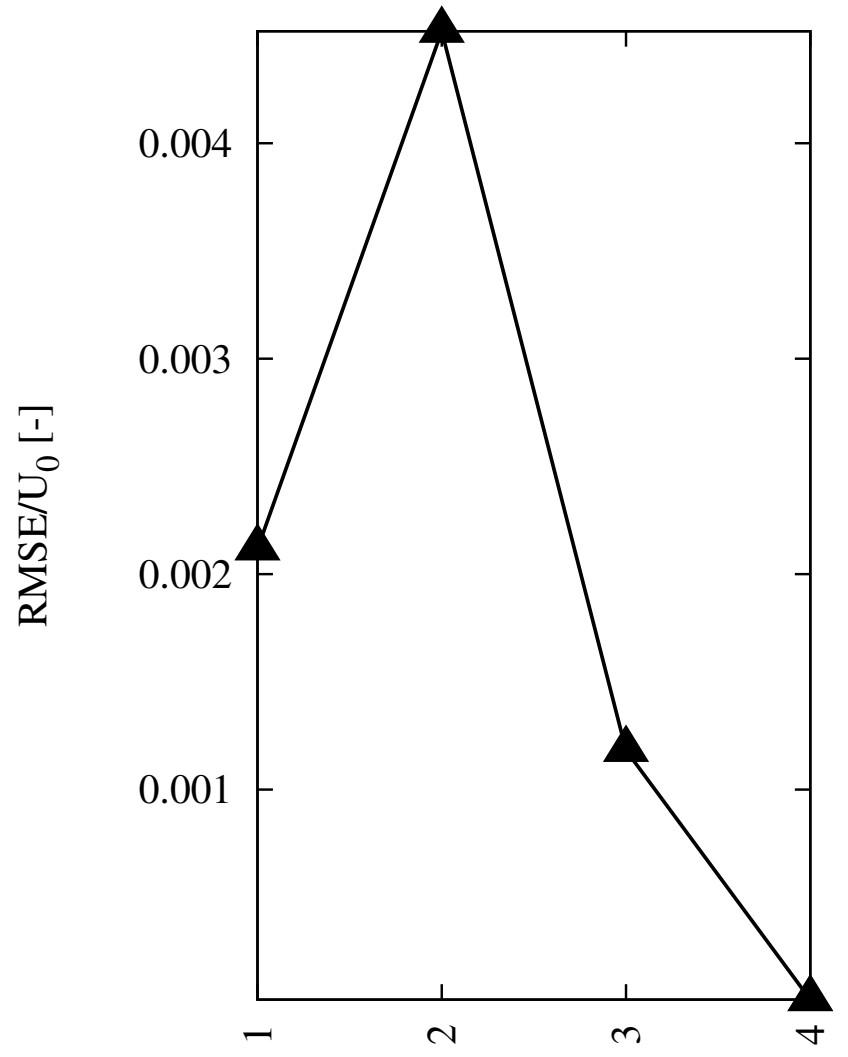

Refinement step [-] 
Figure 3 Comparison of simulated velocities $U_{x}$ using different turbulence models with experimental data for flow over a two-dimensional hill, (a) $U_{x}$ velocities at $x_{-03}=-0.30 \mathrm{~m}$, (b) $U_{x}$ velocities at $x_{00}=0.00 \mathrm{~m}$, (c) $U_{x}$ velocities at $x_{01}=0.03 \mathrm{~m}(\mathrm{~d}) U_{x}$ velocities at $x_{02}=0.05 \mathrm{~m}$
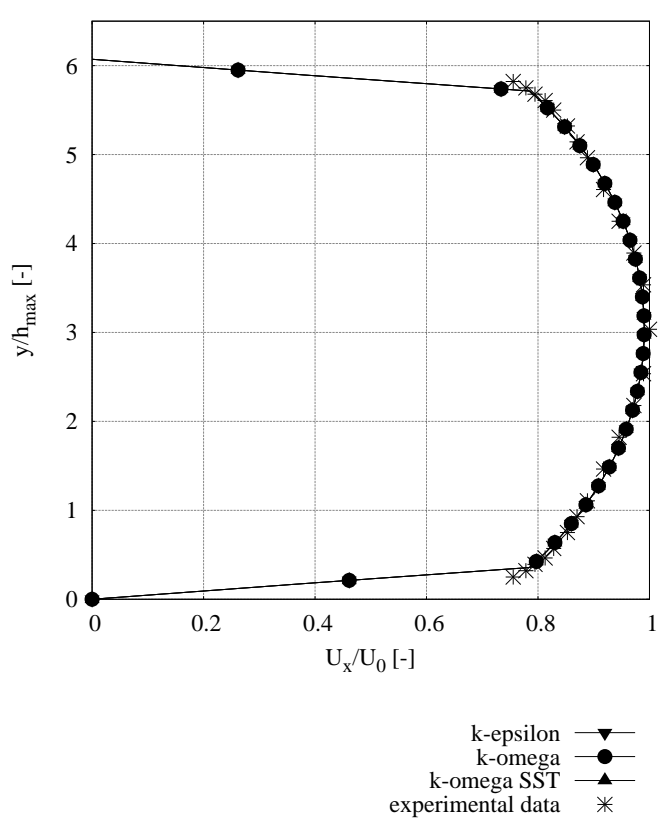

(a)

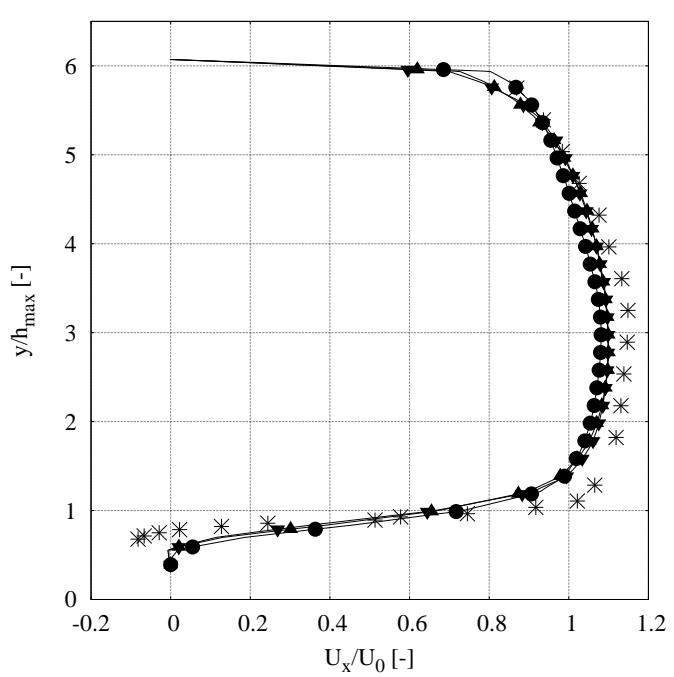

$$
\begin{array}{r}
\text { k-epsilon } \\
\text { k-omega } \\
\text { k-omega SST } \\
\text { perimental data }
\end{array}
$$

(c)
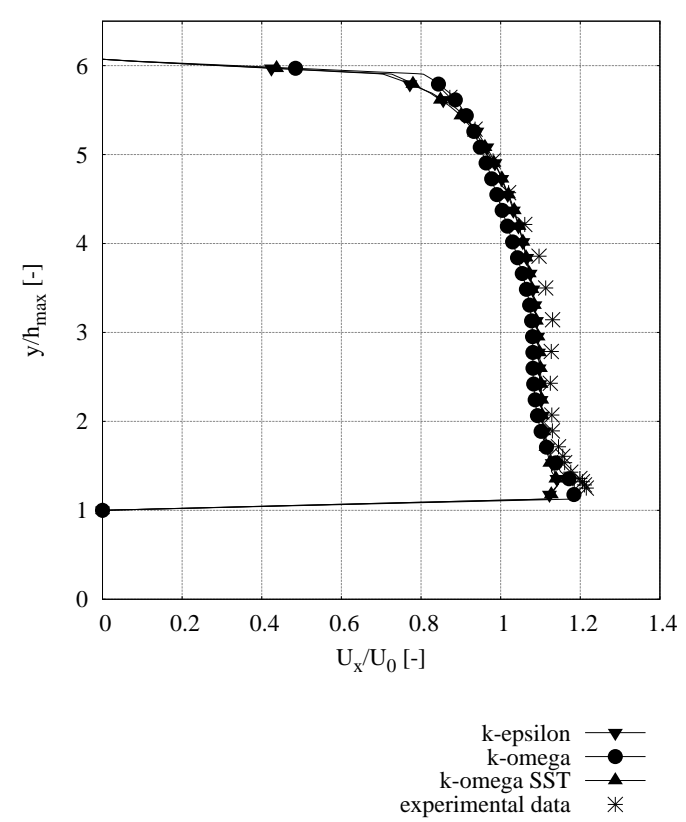

(b)
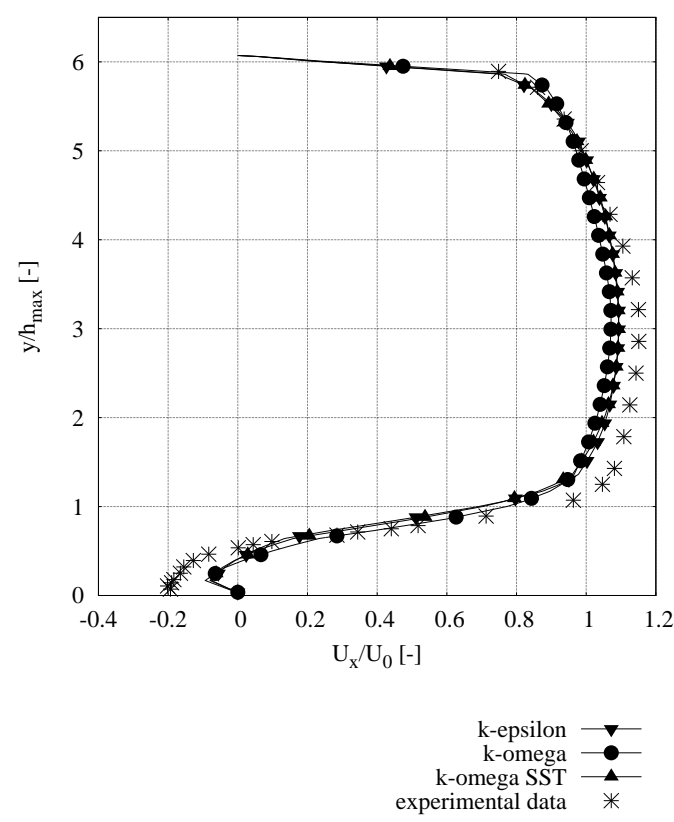

(d) 
Figure 4 Comparison of simulated velocities $U_{y}$ using different turbulence models with experimental data for flow over a two-dimensional hill, (a) $U_{y}$ velocities at $x_{00}=0.00 \mathrm{~m}$ (b) $U_{y}$ velocities $x_{01}=0.03 \mathrm{~m}$
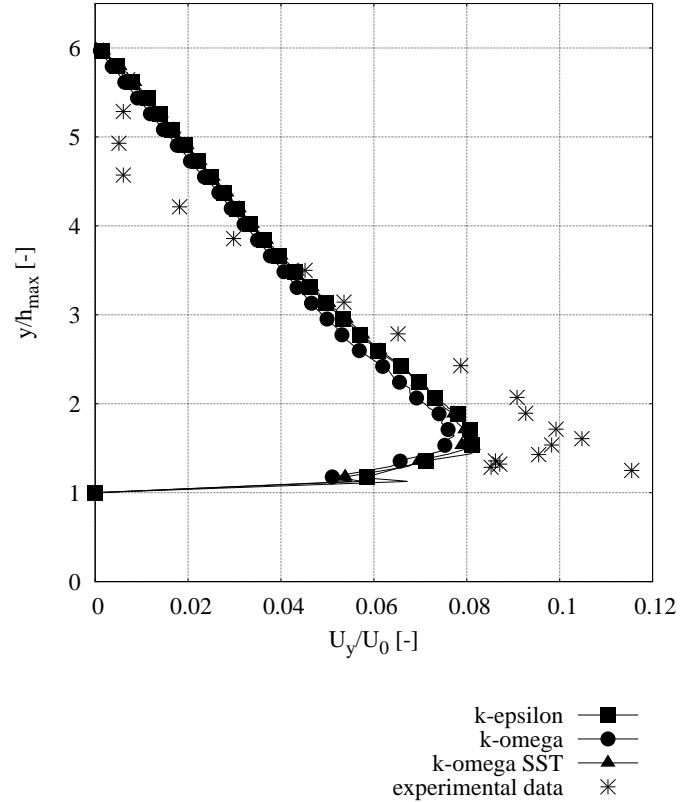

(a)

Table 1 Water phase validation - RMSE/U [-] between experimental results and simulation

\begin{tabular}{lccc}
\hline Observation point & $k-\epsilon$ & $k-\omega$ & $k-\omega S S T$ \\
\hline$U_{x}$ & & & \\
$x_{-03}$ & 0.0515 & 0.0515 & 0.0515 \\
$x_{00}$ & 0.0232 & 0.0307 & 0.0237 \\
$x_{01}$ & 0.0595 & 0.1077 & 0.0681 \\
$x_{02}$ & 0.0361 & 0.0637 & 0.0361 \\
$U_{y}$ & & & \\
$x_{00}$ & 0.0116 & 0.0131 & 0.0130 \\
$x_{01}$ & 0.0156 & 0.0309 & 0.0193 \\
\hline
\end{tabular}

Simulated and measured velocity profiles are compared in Figures $3\left(U_{x}\right)$ and $4\left(U_{y}\right)$. On the horizontal axis, the velocities are displayed in relation to the average flow velocity $U_{0}$. The height on the vertical axis is displayed in relation to the channel height $h_{\max }$. The imposed velocity profile at the inlet of the domain differs slightly from the experimental results of the freestream profile [Figure 3(a)]. Figures $3(\mathrm{~b})$ to $3(\mathrm{~d})$ show the resulting velocity profiles in the reach of the hill structure and enable a qualitative analysis of the accuracy of the different turbulence models. Small deviations can be found between simulations and experimental results. The resulting root mean square error (RMSE) normalised by $U_{0}$ for the different locations is listed in Table 1. The error has been calculated using linear interpolation between the cell values of the simulation results. Overall, the Standard k- $\epsilon$ turbulence model leads to slightly better results in all locations than the other RANS models analysed. The velocities in y-direction $\left(U_{y}\right)$
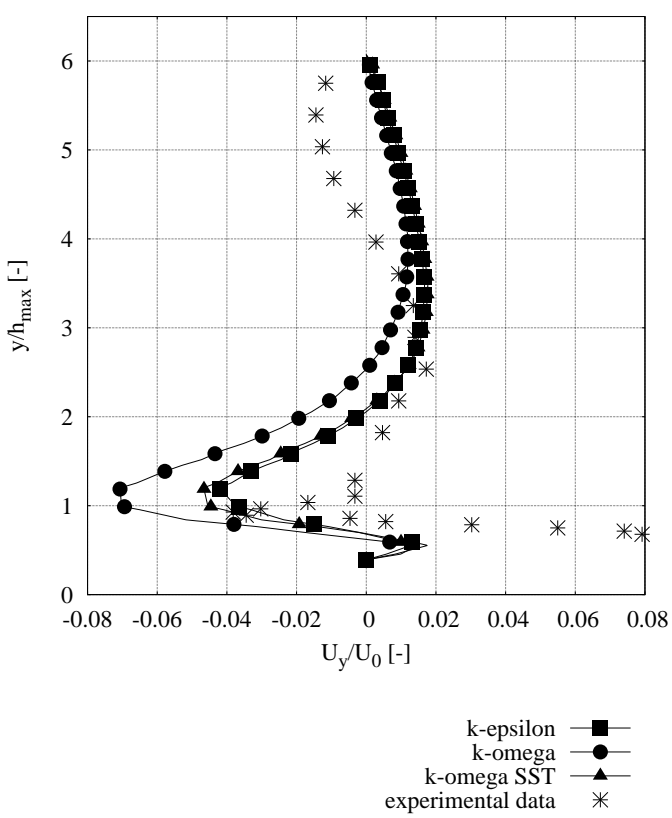

(b)

show similar results (Figure 4). Table 1 shows that the error between the experimental results and the simulated cases is smaller than the error of the $U_{x}$-velocities. In comparison to the velocities in $\mathrm{x}$-direction, the velocities in $y$-direction are very small, being more challenging for numerical simulations and measuring.

It can be concluded, that the chosen model set-up is able to describe a relatively complex hydraulic test case for a single-phase flow problem appropriately and all turbulence models show a good accuracy. Due to the good performance in this case as well as in the cases referred to in Section 1, the Standard $\mathrm{k}-\varepsilon$ turbulence model will be used in the following cases.

\subsection{Free surface flow over a hill}

After having analysed the water phase behaviour, a two-phase flow has been simulated in order to investigate the water surface behaviour. The model setup was slightly changed. A free surface flow was modelled in a two-dimensional model domain. Again, the system was bounded by upper and lower walls. Under subcritical flow conditions the water level drawdown was analysed and compared to the analytically computed water level deviation by using continuity and Bernoulli's equation. The computation time was approximately $1 \mathrm{~h}$ for parallel computation on 16 cores. In the following, three cases are investigated (Table 2). The two-dimensional model domains of cases 1 and 2 consisted of 68,542 cells with a minimum cell length of $0.0024 \mathrm{~m}$ and a maximum cell length of $0.15 \mathrm{~m}$. The domain of case 3 was discretised into 
175,762 cells. The cell length ranged between $0.0035 \mathrm{~m}$ and $0.144 \mathrm{~m}$. A time step of $\Delta \mathrm{t}=0.001 \mathrm{~s}$ was chosen.

Table 2 Water-air-interface - properties of the different test cases

\begin{tabular}{lccc}
\hline Class & Case 1 & Case 2 & Case 3 \\
\hline Length of domain & $25 \mathrm{~m}$ & $25 \mathrm{~m}$ & $35 \mathrm{~m}$ \\
Height of domain & $2 \mathrm{~m}$ & $2 \mathrm{~m}$ & $6 \mathrm{~m}$ \\
$h_{1}$ & $1.0 \mathrm{~m}$ & $1.0 \mathrm{~m}$ & $3.0 \mathrm{~m}$ \\
$v_{1}$ & $1.0 \mathrm{~m} / \mathrm{s}$ & $1.25 \mathrm{~m} / \mathrm{s}$ & $3.0 \mathrm{~m} / \mathrm{s}$ \\
$\Delta z$ & $0.2 \mathrm{~m}$ & $0.2 \mathrm{~m}$ & $0.2 \mathrm{~m}$ \\
$\Delta h$, analytical & $0.036 \mathrm{~m}$ & $0.070 \mathrm{~m}$ & $0.110 \mathrm{~m}$ \\
$\Delta h$, numerical & $0.042 \mathrm{~m}$ & $0.090 \mathrm{~m}$ & $0.140 \mathrm{~m}$ \\
\hline
\end{tabular}

The boundary conditions were defined as follows: the inlet was divided in two parts, containing an inlet for the air phase and an inlet for the water phase. The air-inlet was specified with a fixed total pressure, the water-inlet was specified with a fixed discharge. Since the analysed test cases are two-dimensional, sidewalls were defined as empty boundaries. The upper and lower walls were specified using no-slip conditions. The outlet was defined using the pressure boundary condition mentioned in Section 2.3. As initial conditions for the pressure and velocity, the freestream velocities $\left(v_{1}\right)$ and water levels $\left(h_{1}\right)$ as listed in Table 2 have been defined.

In the following, the results of different two-phase flow simulations for three different model setups are presented. Variations were made concerning the discharge and water level. A deeper analysis of factors such as the structure of the sill and the flow regime can be found in Teuber et al. (2016).

In the three cases presented here, the water depth and flow velocity have been varied using the two-dimensional test case with a $30^{\circ}$ angular structure of the ground sill (Figure 5), the flow regime was kept subcritical. Information about the setup including the analytically calculated water level drawdown and the numerically simulated water level drawdown are listed in Table 2, where $v_{1}$ is the approaching velocity and $h_{1}$ the water depth in front of the sill. The domain of case 1 is displayed in Figure 5. The results show a similar trend between the simulated results and the analytical solution. The reason for the higher drawdown obtained by the numerical solution are additional energy losses due to the structure of the sill that can be accounted for by the CFD simulation whereas the analytical solution using continuity and Bernoulli's equation leads to a one-dimensional solution that neglects single energy losses caused by the structure of the sill. In addition, Teuber et al. (2016) simulated a strictly supercritical setup leading to a rise of the water level above the sill. In agreement with the previous observations, the water level deviation coincided with the analytical solution.

It can be concluded that all the cases investigated show that the numerically calculated deviation of the water level reasonably coincides with the analytical solution.

\subsection{Complex free surface flow in a sewer model}

As a third step, the overall performance of the chosen model setup was tested using the complex sewer geometry of Bayón et al. (2015). Data from an existing CFD model as well as measured data gained from a 1:20 scale model has been compared to simulations using a model setup describing a closed duct bounded by upper and lower walls as well as sidewalls. The simulation results reported in Bayón et al. (2015) were obtained using a similar configuration as presented in this paper, also implemented in OpenFOAM with the difference in the definition of a simplified top and outlet boundary condition. The Standard $\mathrm{k}-\epsilon$ model was used as closure to the turbulent stresses in both models.

The model domain describes a planned sewer stretch which has been developed due to a necessary diversion of an existing sewer stretch in València, Spain (Figure 6). The chosen stretch has a length of approx. $95 \mathrm{~m}$ and consists of an initial stretch, which was modelled in order to achieve fully developed flow conditions. After the initial stretch, a $45^{\circ}$ constant radius curve and a straight stabilisation stretch with a length of $10 \mathrm{~m}$ follow. Having passed the curve, the water then reaches a spillway followed by a stilling basin which is designed in order to control the occurrence of critical flow conditions, obtain a smooth change of slope between the existing and new sewer stretch and to expand the channel width from $6.0 \mathrm{~m}$ to $7.5 \mathrm{~m}$. An adjacent transition zone leads the flow into the ovoid channel with a length of $20 \mathrm{~m}$ which is the last part of the section. In order to force the flow back to a subcritical state before reentering into the ovoid section, macro-roughness elements are placed in the stilling basin and a hydraulic jump is forced. Due to its different features as well as the different flow regimes, the chosen geometry leads to a model domain that is highly complex in terms of hydraulic behaviour.

The innovation of the simulations presented in this paper compared to the existing CFD simulations carried out by Bayón et al. (2015) is that here the whole domain was considered as a closed duct leading to a realistic top boundary condition and another outflow boundary condition to ensure stability. As mentioned before, the same case has been previously simulated by Bayón et al. (2015) with a simplification for the closed conduit, i.e., an atmospheric top boundary in order to avoid stability problems. In this paper, the top boundary was not described with an atmospheric condition but as a wall with no-slip condition. The lower walls as well as the sidewalls were defined using no-slip conditions in both cases. The remaining boundary conditions were defined as follows by Bayón et al. (2015): the outlet water level was set to $5.01 \mathrm{~m}$ and the discharge was defined as $100 \mathrm{~m}^{3} / \mathrm{s}$. Accordingly, the pressure and velocity outlet boundary condition were defined. At the outlet, the velocity was defined to a constant value which forced the water level to a certain height. Within the domain, an initial water level and flow velocity were defined according to these values. 
Figure 5 Free surface flow over a hill - model domain of case 1 (see online version for colours)

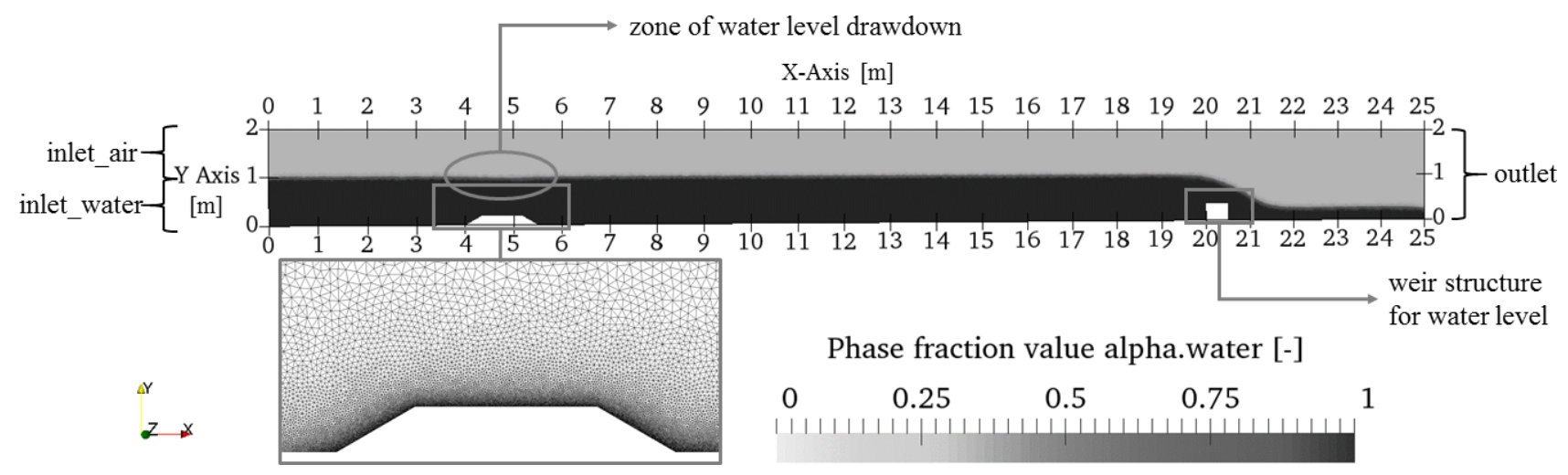

Figure 6 Complex sewer geometry - geometry of the sewer stretch

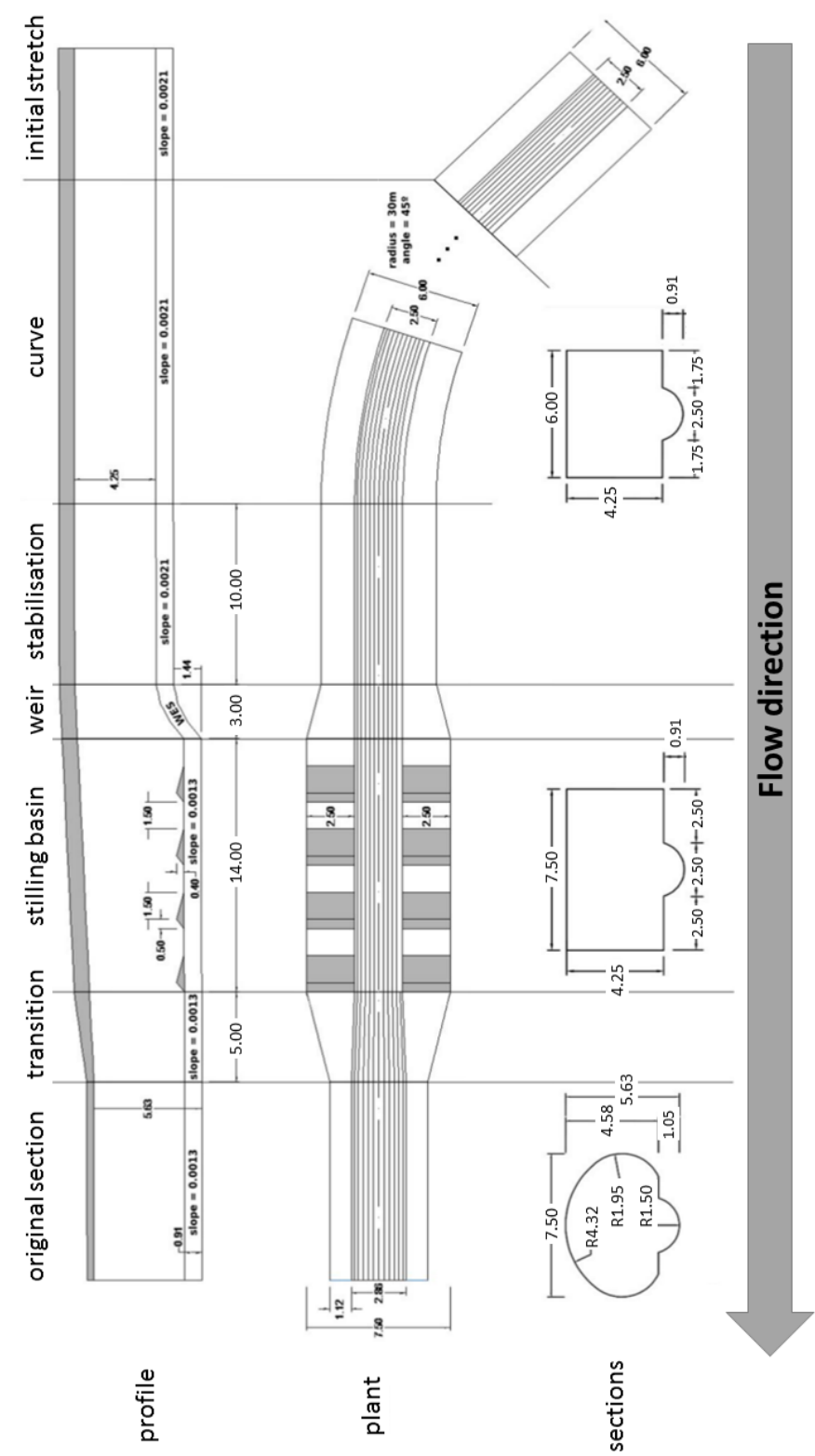

Source: Bayón et al. (2015) 
Figure 7 Complex sewer geometry - overview of monitoring points for water surface profiles (see online version for colours)
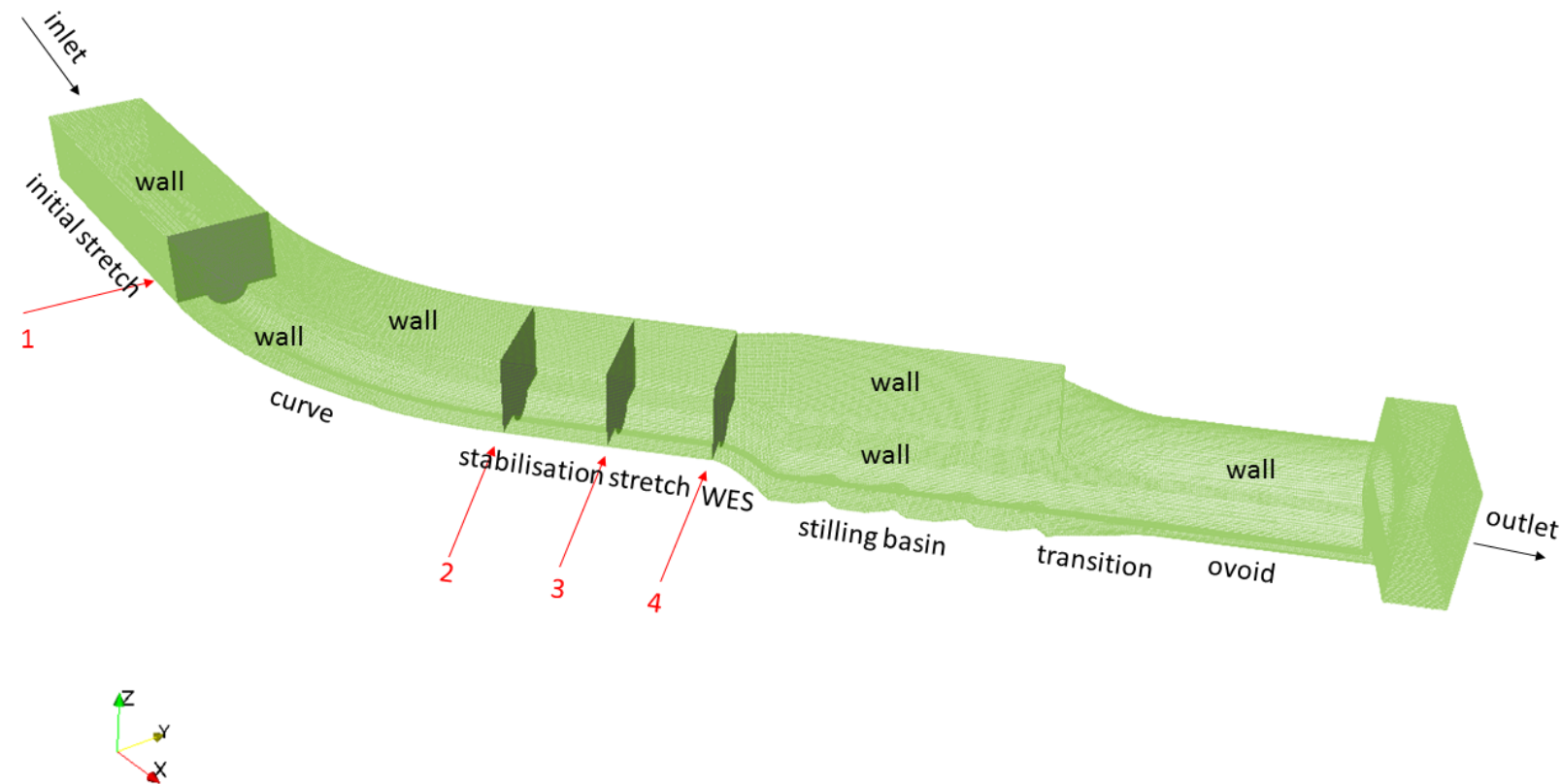

Figure 8 Complex sewer geometry - comparison of water surface profile at different locations (see online version for colours)

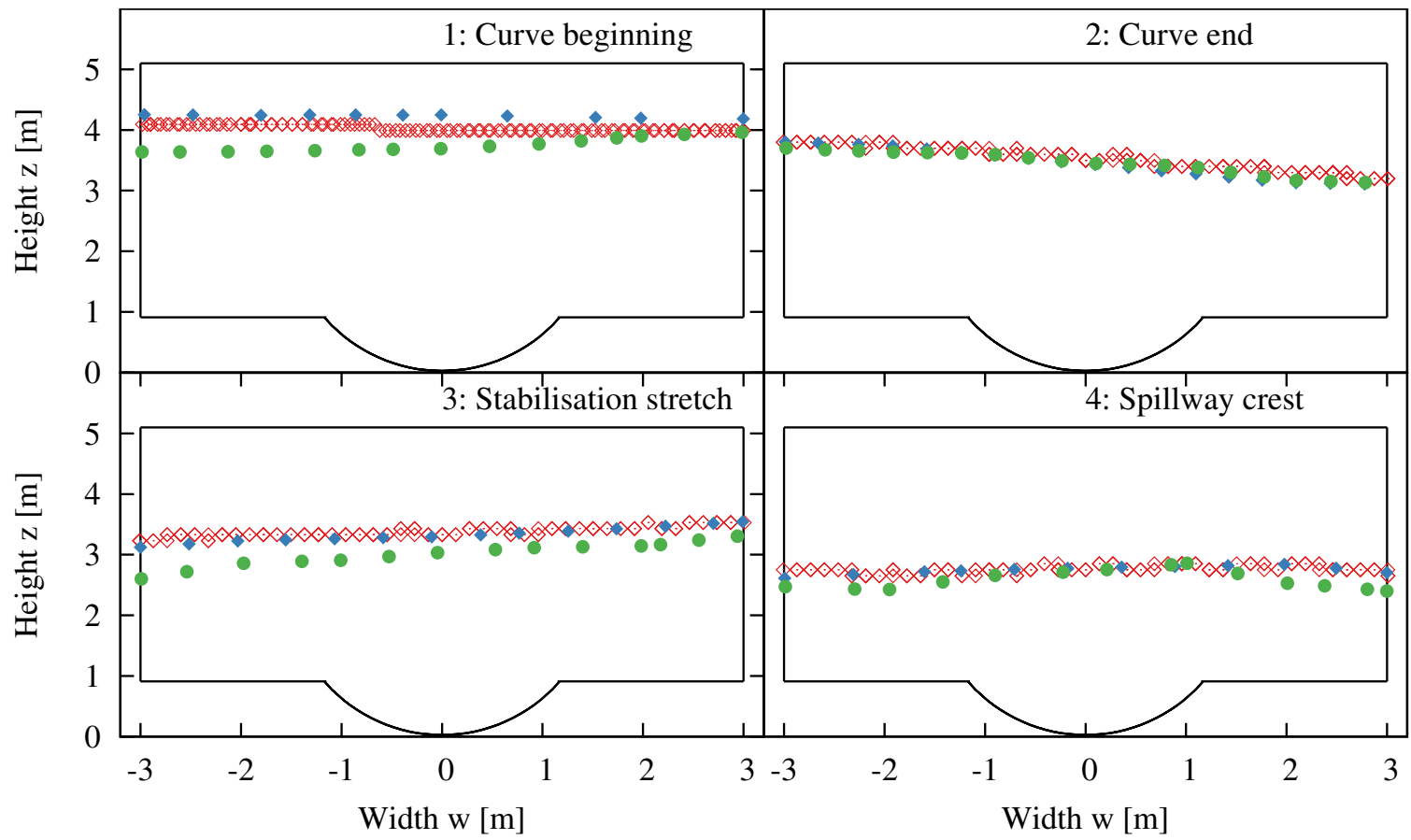

Physical model $\quad$ CFD-model with weir (new)

CFD-model (existing)

Note: Existing CFD model - results obtained by Bayón et al. (2015); new CFD model - results obtained in this paper. 
In this paper, a weir structure has been added in close proximity to the outlet in order to obtain the desired water level. This setup is very robust concerning initial conditions and does not necessarily need a correct initialisation of water level and flow velocity. Stable simulations can even be obtained under dry initial conditions. In the following, the simulation results using the weir outlet boundary condition will be compared to experimental measurements as well as to existing CFD simulations as they have been carried out by Bayón et al. (2015).

In Bayón et al. (2015) the accuracy of different mesh sizes ranging from $0.0861 \mathrm{~m}$ to $0.1236 \mathrm{~m}$ were compared. Mesh convergence has been reached for a mesh size of $0.103 \mathrm{~m}$. In this paper, simulations were performed on a grid with the same resolution, leading to an overall number of $3,029,223$ cells. The simulations demanded a computation time of $336 \mathrm{~h}$ on 96 cores. Simulations were performed with an adjustable time step in relation to the Courant number which converged against $\Delta \mathrm{t}=0.0002 \mathrm{~s}$. The simulations run stable on different grid sizes, even if the domain is dry in the beginning of the simulations.

In order to compare the accuracy of the results for this complex test case, the water level distribution at four different cross sections has been compared. Figure 7 shows the location of the different monitoring points (one - beginning of curve, two - end of the curve, three stabilisation stretch, four - spillway crest). The resulting water surface profiles in the different cross sections of the two CFD models and the physical model are displayed in Figure 8 . The results obtained in this paper are called 'new CFD model' in the following, while the results of Bayón et al. (2015) are called 'existing CFD model'. A qualitative comparison shows an overall good agreement of the new CFD model with the existing model. In the beginning of the curve, the water level is closer to the physical model than the existing CFD model. In the remaining monitoring points, the results of the new CFD model are similar to the results of the existing model. This leads to the conclusion that the new CFD model with a realistic top boundary condition is as accurate as the existing model using a simplified boundary condition (atmospheric top boundary).

The results show that the closed model setup consisting of a weir structure as outlet boundary condition, a high-resolution mesh and the Standard k- $\varepsilon$ turbulence model is stable concerning initially dry conditions, flow transitions from super to subcritical flow as well as high filling ratios and leads to reliable results.

\section{Conclusions}

The aim of this study was to investigate OpenFOAM's two-phase solver interFoam concerning its ability to accurately describe complex hydraulic test cases in closed ducts when a certain outlet water level is given. This paper presents three different application cases where the interFoam solver has been used. Another aim was to evaluate the accuracy and suitability of different RANS turbulence models.
In a first step, a single-phase water flow over a hill has been simulated and different RANS turbulence models have been validated concerning their accuracy in describing the eddy structure behind the hill. The flow velocities measured experimentally were successfully reproduced by the different models. In the following, the Standard $\mathrm{k}-\varepsilon$ turbulence model has been used for test cases describing the water surface behaviour under quasi-steady state conditions. As second test case, free surface flow over a hill has been simulated. Different parameters such as the discharge and the water level have been varied and the numerically computed results were compared to an analytical solution obtained by using continuity and Bernoulli's equation. The simulations were able to predict a similar trend of the water level drawdown compared to the analytical solution. Due to additional energy losses caused by the specific structure of the sill, the analytically calculated drawdown is smaller than the CFD simulations but reasonably coincides for different simulations. These results can be seen to support the adequacy of the applied numerical modelling. In a third step, complex free surface flow in a sewer model has been analysed in order to investigate the stability of the simulations using the setup of a complex geometry. A comparison with results of an existing CFD model, which used a simplified top boundary condition (atmospheric instead of closed) and another outflow boundary condition, as well as measured results from a 1:20 scale model showed a good agreement of this setup with the existing results.

Summing up, the VOF approach implemented in OpenFOAM is capable of describing complex two-phase flows in closed ducts. Small differences in the accuracy have been observed depending on the chosen turbulence models. The possibility of simulating even complex closed systems opens up the chance to describe complex phenomena such as odour and corrosion caused by hydrogen sulphide in concrete sewers. Future research will focus on the detailed behaviour of the air phase.

\section{Acknowledgements}

Parts of the simulations were computed on the supercomputers of Norddeutscher Verbund für Hoch- und Höchstleistungsrechnen in Berlin.

\section{Funding}

The funding provided by the German Research Foundation (DFG) within the Research Training Group 'Urban Water Interfaces' (GRK 2032) is gratefully acknowledged.

\section{References}

Almeida, G., Durao, D.F.G. and Heitor, M.V. (1993) 'Wake flows behind two-dimensional model hills', Experimental Thermal and Fluid Science, Vol. 7, No. 1, pp.87-101. 
Bayón, A., Vallés-Morán, F.J. and López-Jiménez, P.A. (2015) 'Numerical analysis and validation of South Valencia sewage collection system diversion' in E-Proceedings of the 36th IAHR World Congress, The Hague, The Netherlands.

Bayón, A. and López-Jiménez, P.A. (2015) 'Numerical analysis of hydraulic jumps using OpenFOAM', Journal of Hydroinformatics, Vol. 4, No. 17, pp.662-677.

Berberović, E., van Hinsberg, N.P., Jakirlić, S., Roisman, I.V. and Tropea, C. (2009) 'Drop impact onto a liquid layer of finite thickness: dynamics of the cavity evolution', Physical Review E, Vol. 79, No. 3, p.036306.

Celik, I.B., Ghia, U. and Roache, P.J. (2008) 'Procedure for estimation and reporting of uncertainty due to discretization in CFD applications', Journal of fluids Engineering-Transactions of the ASME, Vol. 130, No. 7, pp.780011-780014.

Cifani, P., Michalek, W.R., Priems, G.J.M, Kuerten, J.G.M. and van der Geld, C.W.M. (2016) 'A comparison between the surface compression method and an interface reconstruction method for the VOF approach', Computers and Fluids, Vol. 136, pp.421-435.

Davroux, A. , Hoa, C. and Laurence, D. (2014) 'Flow over a $2 \mathrm{D}$ hill-reference solutions for $\mathrm{k}-\varepsilon$ and second moment closure turbulence models', 4th ERCOFTAC Workshop on Turbulence Modelling, Karlsruhe, Germany.

Edwini-Bonsu, S. and Steffler, P.M. (2004) 'Air flow in sanitary conduits due to wastewater drag: a computational fluid dynamics approach', Journal of Environmental Engineering Science, Vol. 3, No. 5, pp.331-342.

Gessner, M.O., Hinkelmann, R., Nützmann, G., Jekel, M., Singer, G., Lewandowski, J., Nehls, T. and Barjenbruch, M. (2014) 'Urban water interfaces', Journal of Hydrology, Vol. 514, pp.226-232.

Greenshields, C.J. and CFD Direct (2015) OpenFOAM User Guide Version 2.4.0, OpenFOAM Foundation Ltd., London, UK.

Higuera, P., Lara, J.L., and Losada, I.J. (2013) 'Simulating coastal engineering processes with OpenFOAM ${ }^{\circledR}$, , Coastal Engineering, Vol. 71, pp.119-134.

Hirt, C.W. and Nichols, B.D. (1981) 'Volume of fluid (VOF) method for the dynamics of free boundaries', Journal of Computational Physics, Vol. 39, No. 1, pp.201-225.

Hoang, D.A., van Steijn, V., Portela, L.M., Kreutzer, M.T. and Kleijn, C.R. (2013) 'Benchmark numerical simulations of segmented two-phase flows in microchannels using the volume of fluid method', Computers and Fluids, Vol. 86, pp.28-36.

Hvitved-Jacobsen, T., Vollertsen, J. and Nielsen, A.H. (2013) Sewer Processes: Microbial and Chemical Process Engineering of Sewer Networks, 2nd ed., CRC Press, Boca Raton, USA.

Issa, R.I. (1985) 'Solution of the implicitly discretized fluid flow equations by operator splitting', Journal of Computational Physics, Vol. 62, No. 1, pp.40-65.

Jasak, H. (1996) Error Analysis and Estimation for the Finite Volume Method with Applications to Fluid Flows, PhD thesis, Imperial College London (University of London), United Kingdom.

Keough, S. (2014) Optimising the Parallelisation of OpenFOAM Simulations, $\mathrm{PhD}$ thesis, Australian Government Department of Defense, Australia.
Kinyua, M.N., Zhang, J., Camacho-Céspedes, F., Tejada-Martinez, A. and Ergas, S.J. (2016) 'Use of physical and biological process models to understand the performance of tubular anaerobic digesters', Biochemical Engineering Journal, Vol. 107, pp.35-44.

Launder, B.E. and Sharma, B.I. (1974) 'Application of the energy dissipation model of turbulence to the calculation of flow near a spinning disc', Letters in Heat and Mass Transfer, Vol. 1, No. 2, pp.131-137.

Marschall, H. (2011) Towards the Numerical Simulation of Multi-Scale Two-Phase Flows, $\mathrm{PhD}$ thesis, Technische Universität München, München.

Márquez Damián, S. (2012) Description and Utilization of Interfoam Multiphase Solver, Report.

Márquez Damián, S. (2013) An Extended Mixture Model for the Simulaneous Treatment of Short and Long-scale Interfaces, $\mathrm{PhD}$ thesis, Universidad Nacional Del Litoral, Argentina.

Menter, F.R.(1993) 'Zonal two-equation k- $\omega$ turbulence models for aerodynamic flows', in 23rd Fluid Dynamics, Plasmadynamics, and Lasers Conference, Orlando, USA, p.2906.

Menter, F.R.(1994) 'Two-equation eddy-viscosity turbulence models for engineering applications', AIAA Journal, Vol. 32, No. 8, pp.1598-1605.

Patankar, S. and Spalding, D (1972) 'A calculation procedure for heat, mass and momentum transfer in three-dimensional parabolic flows', Journal of Heat and Mass Transfer, Vol. 15 , pp.1787-1806.

Rootsey, R., Melchers, R., Stuetz, R., Keller, J. and Yuan, Z. (2012) 'Taking control of odours and corrosion in sewers', in Proceedings of the National Water Conference and Exhibition (OzWater 2012), Sydney, Australia.

Rusche, H. (2002) Computational Fluid Dynamics of Dispersed Two-phase Flows at High Phase Fractions, PhD thesis, Imperial College London (University of London), UK.

Schulze, L. and Thorenz, C. (2014) 'The multiphase capabilities of the CFD toolbox OpenFOAM for hydraulic engineering applications', in Proceedings of the 11th International Conference on Hydroscience and Engineering (ICHE), Hamburg, Germany.

Shuard, A.M., Mahmud, H.B. and King, A.J. (2016) 'Comparison of two-phase pipe flow in OpenFOAM with a mechanistic model', IOP Conference Series: Materials Science and Engineering, Vol. 121, No. 1.

Teuber, K., Broecker, T., Barjenbruch, M. and Hinkelmann, R. (2016) 'High-resolution numerical analysis of flow over a ground sill using OpenFOAM' in Proceedings of the 12th International Conference on Hydroscience and Engineering (ICHE), Tainan, Taiwan.

Thaker, J.P. and Banerjee, J. (2013) 'CFD simulation of two-phase flow phenomena in horizontal pipelines using OpenFOAM', in Proceedings of the 40th National Conference on Fluid Mechanics and Fluid Power, Himachal Pradesh, India.

Thorenz, C. and Strybny, J. (2012) 'On the numerical modelling of filling-emptying systems for locks', in Proceedings of the 10th International Conference on Hydroinformatics (HIC), Hamburg, Germany.

Wilcox, D.C. (1988) 'Re-assessment of the scale-determining equation for advanced turbulence models', AIAA Journal, Vol. 26, No. 11, pp.1299-1310. 